\title{
Subgaleal shunting: A 20-year experience
}

\author{
Martin H. Savitz, M.D., AND Leonard I. Malis, M.D. \\ From the Division of Neurosurgery, Nyack Hospital, and Department of Neurosurgery, Good \\ Samaritan Hospital, Suffern; and the Department of Neurosurgery, The Mount Sinai Hospital, New \\ York, New York
}

\begin{abstract}
Object. The authors undertook a retropective study to evaluate the effectiveness of diverting intracranial fluid into the subgaleal space for temporary absorption by the membranes of the scalp.

Methods. Eighty-one patients were treated over a 20 -year period. There were 22 cases of hypertensive hydrocephalus, 52 cases of acute head trauma, and seven cases of chronic subdural hematoma. The simple surgical technique is described.

Subgaleal shunting provided effective short-term treatment of hydrocephalus and increased intracranial pressure, which was monitored directly in 22 cases of cerebral contusion and edema.

Conclusions. The closed method for drainage of cerebrospinal fluid avoids the complications of open ventriculostomy or open drainage of the subdural space.
\end{abstract}

\section{KEY WORDS • closed head injury • intracranial pressure • cerebral contusion • hydrocephalus • subdural hematoma}

In 1896, von Mikulicz ${ }^{21}$ described a procedure for diverting intracranial fluid into the subgaleal space. The procedure was reviewed by Cushing ${ }^{1}$ in 1928 and Davidoff $^{3}$ in 1929 for the relief of hydrocephalus. Chronic postoperative CSF fistulas were subsequently treated by ventriculosubcutaneous drainage ${ }^{2,6}$ before Perret and Graf ${ }^{13}$ in 1977 reported a method of temporarily decompressing the ventricular system enlarged by tumors and of treating recurrent subdural hematomas by diverting fluid into the parietal subgaleal space. One of the present authors (L.I.M.) $)^{9}$ taught his residents to avoid the use of exteriorized tubes and receptacles to decrease the risk of infection associated with open ventriculostomy. During his training with the author at The Mount Sinai School of Medicine, H. J. Drabkin placed 15 ventriculosubgaleal shunts via a frontal approach in patients with ventricular dilation. ${ }^{4}$ Insertion of a ventriculosubgaleal shunt in acute head trauma was first reported to the New York Society of Neurosurgery by the second author (M.H.S.) in $1981 .{ }^{18}$ The purpose of this paper is to review and evaluate experience with subgaleal shunting over a 20 -year period.

Abbreviations used in this paper: $\mathrm{CSF}=$ cerebrospinal fluid; $\mathrm{CT}=$ computerized tomography; $\mathrm{ICP}=$ intracranial pressure.

\section{CLINICAL MATERIAL AND METHODS}

\section{Hypertensive Hydrocephalus}

Rapid increase in ventricular size with evidence of periventricular lucencies ${ }^{11}$ observed on CT scanning correlated well with increased ICP in seven patients with hemorrhagic intracranial tumors and 15 patients with subarachnoid and intraventricular hemorrhage (Table 1). After a regular trephine opening in the right-sided parietal area, a ventricular catheter was passed into the lateral ventricle, and the proximal end was left in a previously dissected pouch in the subgaleal space. Accurate two-layer closure of the galea and skin was performed with fine sutures to prevent leakage (Fig. 1).

\section{Acute Head Trauma}

No direct measurement of ICP was attempted in 30 cases (Table 2) of severe closed head injury over a 10year period. ${ }^{17}$ In all 30 patients signs of increased ICP and brainstem compression were demonstrated. Cushing's triad (bradycardia, bradypnea, and hypertension) was recorded in each case. There were no focal findings on neurological examination, and no vault fractures were demonstrated on skull x-ray films. Dexamethasone $(100 \mathrm{mg})$ and mannitol $10 \%(500 \mathrm{ml})$ were given intravenously in the 
TABLE 1

Data obtained in 22 cases of hypertensive hydrocephalus

\begin{tabular}{ccccc}
\hline \hline & \multicolumn{4}{c}{ Outcome/Discharge } \\
\cline { 2 - 5 } & Died & $\begin{array}{c}\text { Permanent } \\
\text { Shunt }\end{array}$ & $\begin{array}{c}\text { Rehab } \\
\text { Center }\end{array}$ & Home \\
\hline $\begin{array}{c}\text { Patient Diagnosis } \\
\begin{array}{c}\text { (7 patients) } \\
\text { subarachnoid/intraventricular } \\
\text { hemorrhage (15 patients) }\end{array}\end{array}$ & 0 & 3 & 2 & 5 \\
\hline
\end{tabular}

emergency room. Immediate intubation facilitated hyperventilation, and further evaluation was performed by $\mathrm{CT}$ scanning. Neither hematoma nor midline shift was seen in any of the patients. The ventricles were slitlike (Fig. 2 left), and vesicular hemorrhage in white matter or blood in the ventricular system or subependymal bleeding was visualized (Fig. 2 center).

Using a hand drill, the small ventricles were tapped via a right-sided frontal approach just anterior to the coronal suture. The CSF, obtained under pressure, was 10 to $20 \mathrm{ml}$ in volume and grossly hemorrhagic. A Pudenz ventricular catheter was inserted (Fig. 2 right), and $4 \mathrm{~cm}$ of tubing was tucked into an $8-\mathrm{cm}$ pocket created above the periosteum by dissecting the loose areolar subgaleal tissue. Prophylactic methicillin was administered intravenously every 6 hours. When the Camino ICP monitor became available ${ }^{15}$ the device was inserted into the left frontal subarachnoid space in an additional 22 patients (Table 3 ) prior to placement of a ventriculoperitoneal shunt on the right side (Fig. 3 ). Initial Glascow Coma Scale scores were 3 to 5. The catheter was removed after 7 days without complications.

\section{Chronic Subdural Collections}

Three patients over 50 years of age presented with an acutely obtunded mental status; CT-demonstrated diagnosis of subdural hygromas was made. Because of cerebral atrophy, even in the presence of ventricular enlargement, there was no clear evidence of elevated ICP. In four other patients over 50 years of age in whom chronic subdural hematomas were treated by limited craniectomy and in-

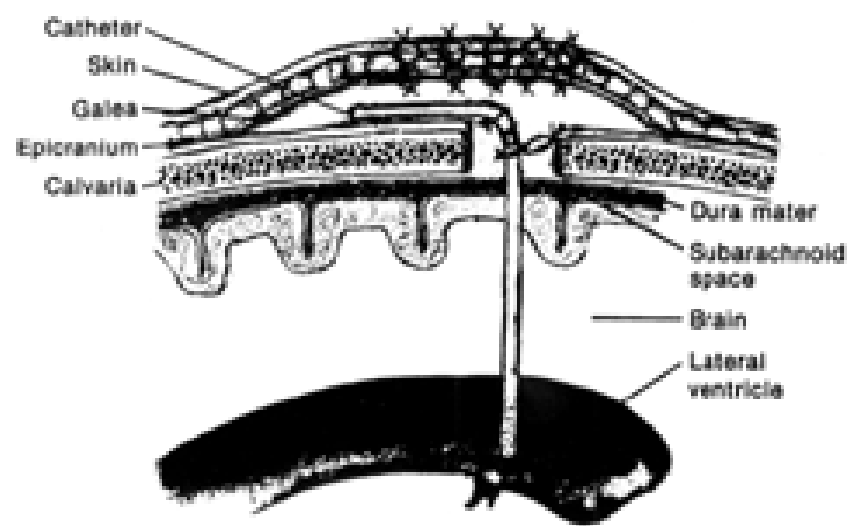

Fig. 1. Artist's drawing showing the ventriculosubgaleal shunt.
TABLE 2

Data obtained in 30 cases of head trauma without ICP monitoring

\begin{tabular}{cccc}
\hline \hline & \multicolumn{3}{c}{ Outcome/Discharge } \\
\cline { 2 - 4 } Grade* $^{*}$ & Died & Rehab Center & Home \\
\hline $\begin{array}{c}\text { Grade III } \\
(24 \text { patients }) \\
\begin{array}{c}\text { Grade IV } \\
(6 \text { patients })\end{array}\end{array}$ & 1 & 18 & 5 \\
\hline
\end{tabular}

* Grading scale presented by L. I. Malis. ${ }^{9}$

sertion of a Jackson-Pratt drain, the reaccumulation of fluid in the subdural space reversed their neurological recovery. All seven patients (Table 4) were treated by placing a Pudenz ventricular catheter in the subdural space. When no craniectomy site was available, a single burr hole was made in the right-sided parietal area with a hand drill. About $4 \mathrm{~cm}$ of tubing was tucked into an $8-\mathrm{cm}$ subgaleal pocket created above the pericranium. The shunts were removed after 10 to 14 days without complications. ${ }^{15}$

\section{RESULTS}

\section{Hypertensive Hydrocephalus}

Eight of the 22 patients died within 72 hours. Temporary drainage of CSF for several weeks provided emergency decompression of the ventricular system with no syphon effect in the other 14 patients. Fibrotic processes progressively stopped the absorption after longer periods. While the subgaleal pocket was soft and fluctuant, simple palpation demonstrated that the ICP was low. If the collection became tense, the galeal and periosteal membranes were no longer able to handle the underlying uncorrected disease. Eight of the 14 patients improved (Table 1). The shorter-length catheter was easily removed for insertion of a ventriculoperitoneal or ventriculoatrial shunt in six cases that had to be converted. There were no complications of wound infection or intraparenchymal hemorrhage.

\section{Acute Head Trauma}

In the first 30 cases in which a shunt was inserted without a monitor (Table 2) as presented by L.I.M., ${ }^{9}$ CT evidence of compressed ventricles and cerebral contusion correlated well with increased ICP. ${ }^{19}$ Six patients categorized as having a Grade IV (no evidence of brain function) in the emergency room did not improve and died within 72 hours. The remaining patients had Grade III function (no eye opening, no verbal response, and motor response limited to abnormal flexion or extension) and were transferred to a rehabilitation center; the exception was a 16year-old boy who suffered a fatal pulmonary embolus on the 30th postoperative day. Each patient was fed via a tube after 3 days of hospitalization. In 12 cases, tracheostomy was required. Subgaleal collections of fluid persisted for 3 to 5 days - the period of brain swelling. The reduction in ICP was evident by immediate improvement in neurological status; hyperventilation, hypertension, and hyperther- 


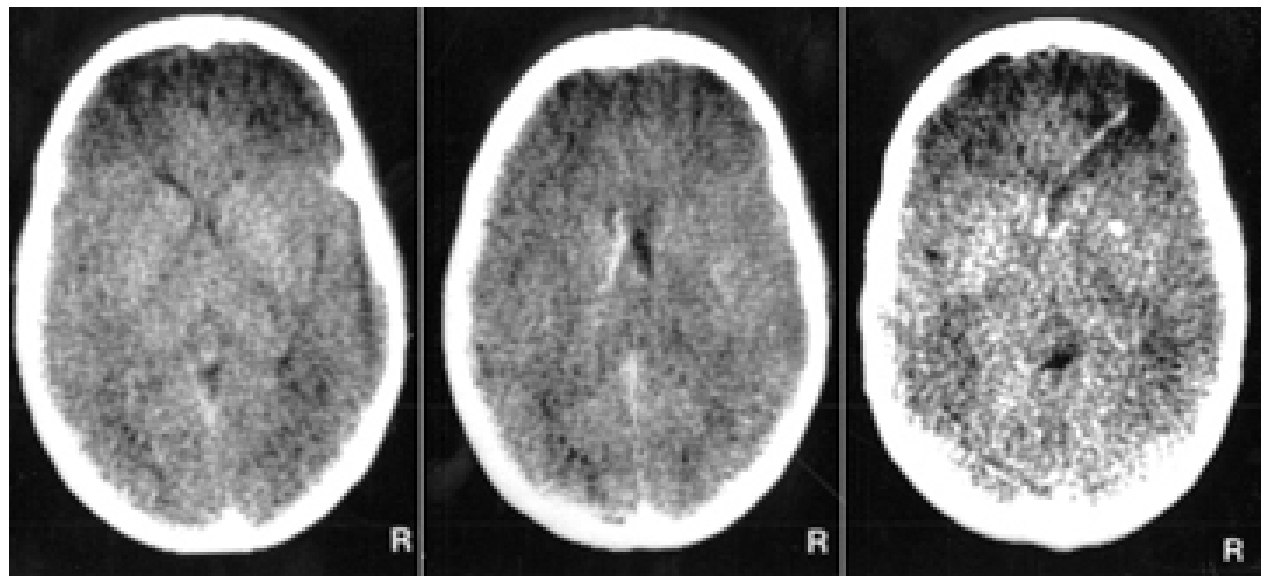

Fig. 2. Computerized tomography scans obtained in patients with acute head trauma. Left: Narrowing of ventricular system can be seen. Center: Note the blood in relation to left lateral ventricle. Right: The catheter is properly positioned. Note the small high-density foci representing areas of cerebral contusion.

mia resolved. In three patients with rhinorrhea CSF stopped leaking from the nose after shunting.

The initial pressure was over $20 \mathrm{~mm} \mathrm{Hg}$ (normal $<10$ $\mathrm{mm} \mathrm{Hg}$ ) in the 22 patients who were monitored (Table 3 ). The recorded ICP in 19 cases remained within normal range for the 72 hours of monitoring after a single intravenous dose of mannitol. Three of the six patients who died had required additional intravenous doses of mannitol to maintain the ICP below $20 \mathrm{~mm} \mathrm{Hg}$. Eight patients required tracheostomy and gastrostomy. One patient regained normal cortical function during her hospitalization for multiple trauma. After 2 to 6 months of rehabilitation and occupational therapy, 15 patients were discharged home in the care of their families. Follow-up CT scans in the six cases in whom brain death was diagnosed within 3 days revealed extensive hemorrhagic infarction (Fig. 4 left) rather than resolution and absorption of areas of contusion (Fig. 4 right). In nine patients with basilar skull fractures CSF ceased leaking after shunting.

\section{Chronic Subdural Collections}

Two of the three patients in whom bilateral subdural hygromas were demonstrated on admission on CT scans had minimal filling of the subgaleal pocket and no neurological improvement. In one of these patients and in all four patients with recurrent subdural hematomas, the sub-

TABLE 3

Data obtained in 22 cases of head trauma with ICP monitoring

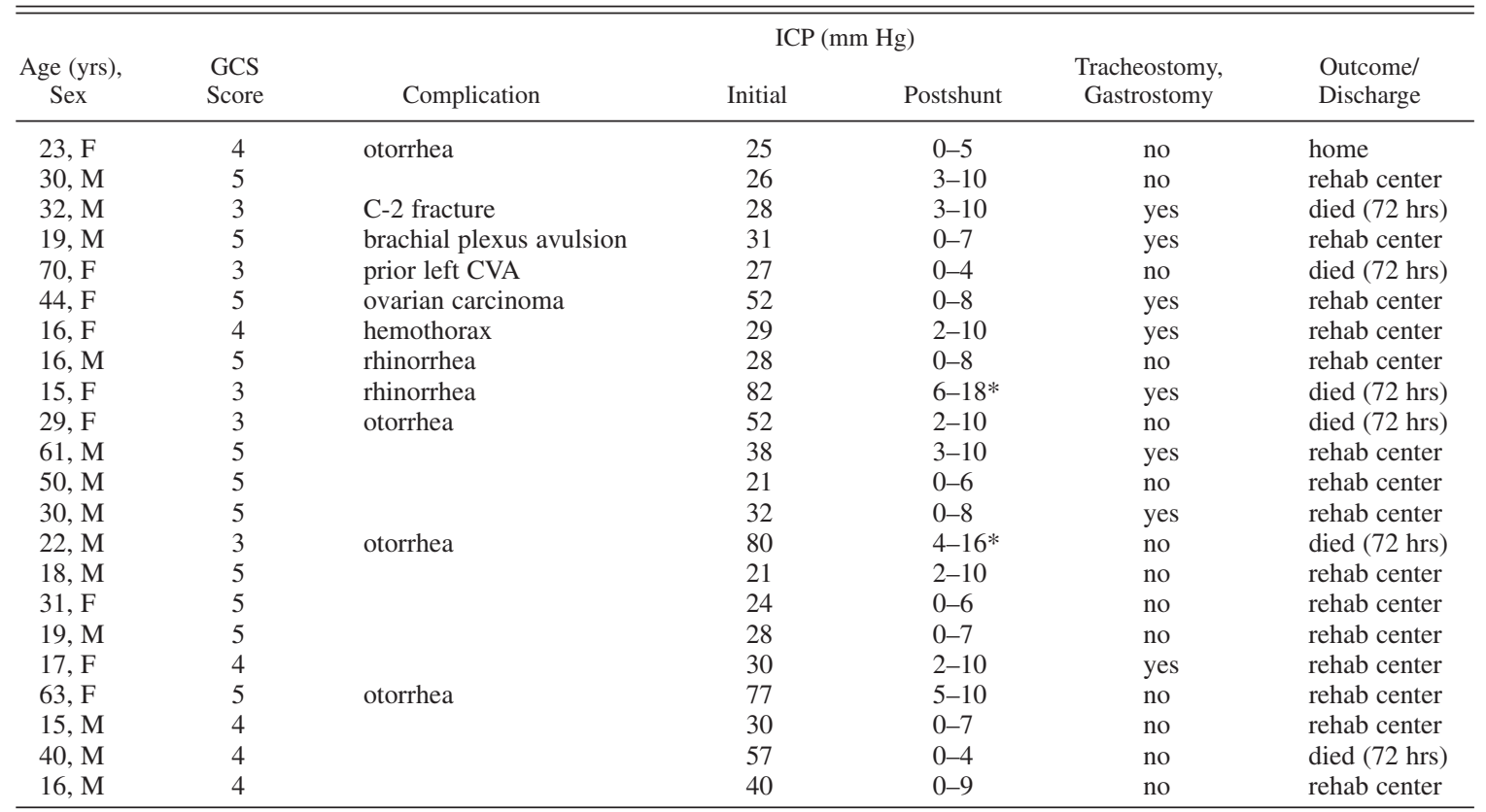

* Insertion delayed due to coagulopathy. Abbreviations: CVA = cerebrovascular accident; GCS = Glasgow coma scale. 


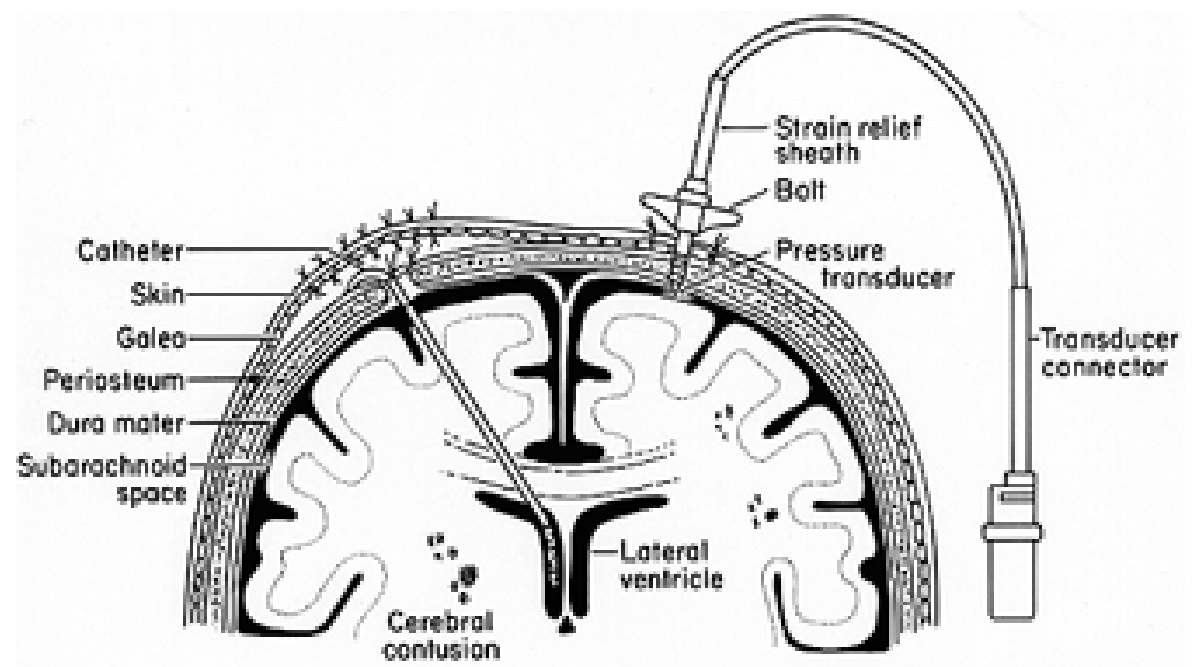

Fig. 3. Artist's drawing showing the ICP monitor in the left frontal area and right subgaleal shunt.

galeal collection remained tense and resolved over a 10 to 14-day period; signs of elevated ICP resolved in all five cases (Table 4).

\section{DISCUSSION}

Perret and Graf ${ }^{13}$ did not list any references following their article in 1977; but in a response ${ }^{14}$ to a letter to the editor written by Ferreira, ${ }^{5}$ they reviewed the prior neurosurgical literature on subgaleal shunts. ${ }^{2,3,6,21}$ Malis, ${ }^{10} \mathrm{com}-$ menting on ventriculosubgaleal shunting and ventriculostomy-related infections in a paper by Holloway, et al., ${ }^{7}$ indicated that open drainage was consistently avoided at The Mount Sinai Hospital over a 25-year period. This practice had been taught by Professor Jean Michel Gybels of Belgium (see Malis ${ }^{9}$ ). In their response, Holloway et al., ${ }^{8}$ while recognizing the advantage of a closed system for ventricular drainage to avoid infection, raised the question of efficacy and the potential for back pressure once the subgaleal pocket had filled.

The absorptive capacity of the subgaleal space has been well documented. ${ }^{3,4,13}$ Inasmuch as high-pressure hydrocephalus was controlled for periods of 3 weeks up to several months, the membranes of the scalp contributed to handling high volume and reducing CSF pressure to normal range. In other patients with intracranial hematomas, midline shifts, and extensive cerebral contusion, leakage into the subgaleal space following decompressive craniectomy could not decrease elevated ICP even with hypnotic, hyperventilation (hypocarbia), hypothermia, and intermittent mannitol therapies. Which of the many factors,

TABLE 4

Outcome in seven cases of chronic subdural hematoma after shunting

\begin{tabular}{lcc}
\hline \hline Shunt Placement & Improved & Unimproved \\
\hline primary bilateral (3 patients) & 1 & 2 \\
secondary unilateral (4 patients) & 4 & 0 \\
\hline
\end{tabular}

such as normal circulation (choroid plexus formation, transependymal absorption, arachnoid villus transport, and elastance) and compliance of the brain, influenced CSF perfusion cannot be determined. ${ }^{12}$

Although attempts to cannulate slitlike ventricles may induce intracerebral bleeding, ${ }^{16}$ no postoperative shunt placement-related hemorrhages were demonstrated on follow-up CT scans. The shunts were removed after 1 week to avoid formation of a subdural hygroma; tracking of fluid around the tubing may occur with or without obstruction of catheters left in place. ${ }^{4,14,20}$ Successful treatment of CSF fistulas by indirect shunting rather than direct repair has been documented in the neurosurgical literature. ${ }^{2,6}$

In the cases requiring shunting of the subdural space, persistence of the subgaleal collection up to 10 to 14 days was consistent with increased ICP, whereas early resolution was diagnostic of a preexisting hygroma. Avoidance of open draining systems eliminated the sequela of tension

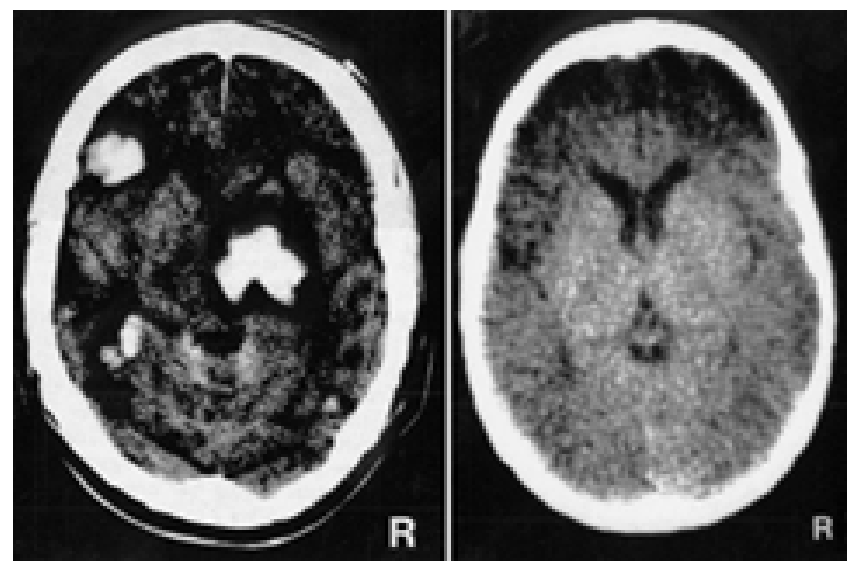

Fig. 4. Computerized tomography scans. Left: Areas of diffuse contusion have coalesced into multiple intracerebral hematomas. Right: The ventricles have returned to normal size and configuration. Note the resolution of contusion. 
pneumocephalus. Perret and Graf ${ }^{13}$ described the use of multiple taps of the subgaleal pocket for diagnostic and therapeutic purposes. Absorption and resolution in the seven patients with chronic subdural collections were monitored only by serial CT scans. ${ }^{15}$

Ventriculosubgaleal shunting can act as a safety valve for continuous reduction of elevated ICP in patients with closed head injuries. The subgaleal space is able to provide adequate drainage and absorption of CSF during the period of traumatic brain swelling. Insertion and removal of the device may be performed rapidly after induction of minimal anesthesia. Avoidance of the exteriorized tubes and receptacles necessary in more elaborate methods of treating increased ICP significantly lowers the risk of infection and can be readily incorporated into the intensive nursing care of comatose patients. Certainly, ICP monitoring over a 72-hour period lends credence to the efficacy of such shunting. The simple method of using the membranes of the scalp for the temporary relief of elevated ICP has broad application in acute head trauma, hypertensive hydrocephalus, and chronic subdural fluid collections.

\section{References}

1. Cushing H: Studies in Intracranial Physiology and Surgery. London: Oxford University Press, 1926, pp 28-29

2. Dardenne G: Le traitment par le drainage ventriculaire souscutane des fistules de liquide cephalo-rachidien postoperatoires rebelles. Acta Neurol Belg 64:1202-1211, 1964

3. Davidoff LM: Treatment of hydrocephalus: historical review and description of a new method. Arch Surg 18:1737-1742, 1929

4. Drabkin AJ, Levine ME, Yang WC: Ventriculo-subgaleal shunt: evaluation by computed tomography. Acta Neurochir 55:107-115, 1980

5. Ferreira NP: Subgaleal shunt for ventricular and subdural drainage. J Neurosurg 49:474-475, 1978 (Letter)

6. Ferreira NP, Correa J: Derivacao liquorica ventriculo-subcutanea na terapeutica cirurgica das fistulas liquoricas pos-operatorias rebeldes. Neurobiologica 35:97-104, 1972
7. Holloway KL, Barnes T, Choi S, et al: Ventriculostomy infections: the effect of monitoring duration and catheter exchange in 584 patients. J Neurosurg 85:419-424, 1996

8. Holloway K, Bullock R, Marmarou A: Ventriculostomy or subgaleal shunting? J Neurosurg 87:486, 1997 (Letter)

9. Malis LI: Acoustic Neuroma. Amsterdam: Elsevier, 1998, p 24

10. Malis LI: Ventriculostomy or subgaleal shunting? J Neurosurg 87:486, 1997 (Letter)

11. Mori K, Murata T, Nakano V, et al: Periventricular lucency in hydrocephalus on computerized tomography. Surg Neurol 8: 337-340, 1977

12. Pappenheimer JR, Heisey SR, Jordan EF, et al: Perfusion of the cerebral ventricular system in unanesthetized goats. Am J Physiol 203:763-774, 1962

13. Perret GE, Graf CJ: Subgaleal shunt for temporary ventricle decompression and subdural drainage. J Neurosurg 47:590-594, 1977

14. Perret GE, Graf CJ: Subgaleal shunt for ventricular and subdural drainage. J Neurosurg 49:474-475, 1978 (Letter)

15. Savitz MH: Another look at ventriculosubgaleal shunting. Mt Sinai J Med 64:189-193, 1997

16. Savitz MH, Bobroff LM: Low incidence of delayed intracerebral hemorrhage secondary to ventriculoperitoneal shunt insertion. J Neurosurg 91:32-34, 1999

17. Savitz MH: Ventriculosubgaleal shunting for acute head trauma: 10 years' experience. J Neurol Orthop Med Surg 12: 205-208, 1991

18. Savitz MH, Katz SS: Ventriculoperitoneal shunting for acute head trauma. Crit Care Med 11:290-293, 1983

19. Tabaddor K, Danziger A, Wisoff HS: Estimation of intracranial pressure by CT closed head trauma. Surg Neurol 18:212-215, 1982

20. Verbeke L, Calliauw L: Cerebrospinal fluid oedema: a rare complication of the ventriculo-subgaleal shunt. Neurochirurgia 30:99-101, 1987

21. von Mikulicz J: Beitrage zur Pathologie und Therapie des Hydrocephalus. Mitteil Grenzgeb Med Chir 1:264-301, 1896

\footnotetext{
Manuscript received September 12, 2000.

Accepted in final form October 4, 2000.

Address reprint requests to: Martin H. Savitz, M.D., 30 Old Phillips Hill Road, New City, New York 10956.
} 